MATEC Web of Conferences 36, 01002 (2015)

DOI: $10.1051 /$ matecconf/ 20153601002

(C) Owned by the authors, published by EDP Sciences, 2015

\title{
Molecular Spectroscopy as an alternative for dating Textiles
}

\author{
Pietro Baraldi ${ }^{1}$, Anna Tinti $^{2}$ \\ ${ }^{1}$ Department of Chemical and Geological Sciences, University of Modena and Reggio Emilia, Via G. Campi, 183, \\ 41125 Modena (Italy) pietro.baraldi@unimore.it \\ ${ }^{2}$ Anna Tinti, Department of Biomedical and Neuromotorial Sciences, University of Bologna, via Belmeloro 8/2, \\ Bologna (Italy)
}

\begin{abstract}
Ancient linen textiles undergo with time a slow change reflected in their Infrared and Raman spectra. The recording of FT-IR and FT-Raman spectra, that reveals a disappearance of some bands and appearance of other ones during the centuries, enables a kinetics of degradation to be followed. Here we discuss the results obtained by using dating methods based on two molecular spectroscopy techniques, that is FT-IR and FT-Raman. A set of samples dating from about 3500 B.C. until present has been examined in order to have an adequate set of reference samples. An accurate analysis of the spectra recorded shows that some differences can be attributed to the evolution of some chemical compounds present in the fibers. A linear correlation between a spectral property and time can be obtained. As an example the date of the Shroud of Turin has been evaluated.
\end{abstract}

Keywords: linen fabric, FT-IR, FT-Raman spectra, dating.

\section{Introduction}

As it is well known [1-6], the organic compounds present in cellulosic fibres change easily with time by losing some particular functional groups, such as small molecules like water and carbon dioxide, and by breaking some chemical bonds or saturating double bonds. This means that, though the concentration of the disappearing groups or of the appearing new ones could be of about few percentages, their evaluation through IR or Raman spectra could be followed. The kinetics of such slow reactions could be followed by evaluating the absorption in correspondence of the maximum of a given band or by calculating the integrated intensity over the whole band. Using samples with an age known from other dating methods, it is possible to build a calibration curve; afterwards, by using statistical methods, it is possible to define the uncertainty of the data obtained from the sample of unknown age. The calibration curve can afterward be used to determine the date of unknown samples. As regards the Shroud of Turin, in a recent book the results obtained by IR and Raman spectroscopies on some fibers withdrawn by it some years ago, have been published [7]. 


\section{Materials and Methods}

For a dating method to be reliably established, some important steps must be followed:

a) the definition and measurement of a property of a fabric as a function of time, with a theoretical background and, as far as possible, independent of factors tied to its conservation history;

b) to dispose of dated samples for tracing a calibration curve between a measured property and time;

c) to evaluate the calibration curve in statistical terms for the evaluation of age from the measured property and its relative accuracy.

One of the major problems is due to factors like temperature and humidity, contamination with external materials, burial and conservation that can change the properties of ancient textiles and therefore influence spectroscopic response to infrared light and Raman scattering. The procedure here adopted exhibit a limited variation of properties if a pre-selection of fibers is done, also on the basis of visual inspection by means of a stereomicroscope and on the sampling technique.

The spreading of data reflected in the correlation coefficients of the resulting equations seems mostly referable to environmental factors that increase variability, but it is still possible to give a rough estimate of dating. Moisture does not seem a detriment in the correlation if a proper textile selection is done. Due to moisture damaging their chemical structure, these fibers are easy to recognize and discharge. Also a heating of flax textiles to relatively high temperature (about $200{ }^{\circ} \mathrm{C}$ for some minutes) can alter their properties, giving a brown hue with the corresponding FT-IR and Raman spectra that can leading to some bias in dating. The comparison of the colour of the sample with other of the same material exposed for different time to various temperatures (e.g. $180^{\circ}, 200^{\circ}, 250^{\circ} \mathrm{C}$ ) and the recording of IR and Raman spectra comparing between themselves can help to establish the method uncertainty in the case under exam [7].

Raman spectra have been recorded with a Bruker Multiram FT-Raman spectrometer, equipped with a cooled Ge-diode detector. The spectral resolution has been set to $4 \mathrm{~cm}^{-1}$ and the total number of scans for each spectrum to 6000 . The excitation source was a Nd3+-YAG laser at $1064 \mathrm{~nm}$ in the backscattering $\left(180^{\circ}\right)$ configuration. All spectra have been recorded with a laser power of $4 \mathrm{~mW}$.

IR spectra have been recorded with a Nicolet 5700 FT-IR spectrometer, equipped with a diamond attenuated total reflectance (ATR) accessory and a DTGS detector, with a spectral resolution of $4 \mathrm{~cm}$ 1. The FT-IR/ATR spectra have been recorded on a single bounce diamond crystal with an area of about $5 \mathrm{~mm}^{2}$, so that the contact size integrates several fibers; the Raman spectra have been recorded on an area of about $0.01 \mathrm{~mm}^{2}$. As the sampling area is relatively small and includes some line fibers, various repeatability and reproducibility tests have been performed.

The evaluation of the response intensity of the spectral parameters was carried out with reference to the cellulose degradation as a first order reaction kinetics, such as the radiocarbon decay. Therefore, in trying to follow the spectroscopic responses by using an exponential law, a correlation coefficient with a natural logarithm treatment of all the data was obtained. We also tested other possible relationships between the variables, in particular by using a linear model or a polynomial interpolation, but the best fitting always resulted the exponential law, confirming the agreement between the kinetic theoretical model and the results obtained. Since the effect of environmental variables, such as temperature and humidity, can change spectral properties, an initial screening was performed in order to choose sufficiently undamaged and not polluted flax fabrics.

As an application of the dating method, the case of the Shroud of Turin has been here considered. Some linen threads coming from a corner were used for the acquisition of both FT-IR/ATR and Raman spectra. 


\section{Spectroscopic dating}

By means of vibrational spectroscopy, two alternative dating methods have been proposed based on FT-IR and Raman analysis. These techniques exhibit different relationships between concentration of a molecular species and the measured parameter. Whereas infrared absorbance follows the LambertBeer's law, and therefore a linear correlation between absorbance and concentration exists, the use of Attenuated Total Reflection (ATR) can significantly change this relation, being both refraction and absorption indexes changing with wavelength and concentration $[13,14]$.

The first method is based on FT-IR ATR spectra. According to the degradation results reported in the literature $[4,8-12]$, many intensity ratios have been examined in order to obtain a correlation of band integrated intensities versus age. The band integrals more sensible to age proved to be those in the range $2600-3080 \mathrm{~cm}^{-1}$ and $3070-3600 \mathrm{~cm}^{-1}$, whereas those in the $800-1180 \mathrm{~cm}^{-1}$ and in the $1500-1760$ $\mathrm{cm}^{-1}$ ranges did not change.

In agreement with Refs. $[14,15]$ two ratios $R_{1}$ and $R_{2}$ of the amplitude peaks integrals of each sample have been evaluated in the corresponding spectra as a function of the wavenumber $\mathrm{n}$ expressed in $\mathrm{cm}^{-1}$ :

$$
\mathrm{R}_{1}=\frac{\int_{2600}^{3080} \mathrm{Idn}}{\int_{1500}^{1760} \mathrm{Idn}} ; \quad \mathrm{R}_{2}=\sqrt{\frac{\int_{2600}^{3080} \mathrm{Idn}}{\int_{800}^{1180} \mathrm{Idn}} \frac{\int_{3070}^{3600} \mathrm{Idn}}{\int_{1500}^{1760} \mathrm{Idn}}}
$$

In Eq. 1 the FT-IR intensity I with a baseline correction, and the integrals $\mathrm{R}_{1}$, and $\mathrm{R}_{2}$ have been taken into consideration to obtain $\mathrm{R}_{\mathrm{C}}$ (a complex index) as a function of wavenumber. $\mathrm{R}_{1}$ expresses the ratio between the intensity of the $\mathrm{CH}$ stretching and $\mathrm{OH}$ bending, whereas $\mathrm{R}_{2}$ depends on the product of $\mathrm{CH}$ and $\mathrm{OH}$ stretching divided by the product of $\mathrm{OH}$ bending and other groups contained in the 800-1180 $\mathrm{cm}^{-1}$ range.

The reference flax samples mentioned in Fig. 1 were tested, of which some have been used for calibration and 3 for testing the fire effects. For example, ratio $R_{1}$ in question with its calibration curve is reported in Fig. 2. The combined ratio $R_{c}[14]$ is reported in Eq. (2) where D is date:

$$
R_{c}=0.5470 e^{0.0003224 D} ; \quad \mathrm{R}=0.9608
$$

that gives

$$
D=1871+3102 \ln R_{c}
$$

In the case of the Shroud of Turin, the FT-IR spectrum is reported in Fig. 3 and $R_{1}=0.291 ; R_{2}=0.365$ were obtained from it [15].

The results on the samples demonstrated that in case of fire effect a bias of some centuries can be observed. Therefore, remembering that the Shroud of Turin was exposed to the 1532 Chamberry fire, the corrected ratios resulted $R_{1}=0.344 ; R_{2}=0.431$. Using equation (2), Ref. [7] obtained by FTIR/ATR analysis on the Shroud's samples a date of 752 B.C. \pm 400 years at a confidence level of $95 \%$, without considering the effect of fire. Taking into account that effect, the authors arrived at a date of 300 B.C. \pm 400 years with a $95 \%$ confidence level. 


\section{MATEC Web of Conferences}

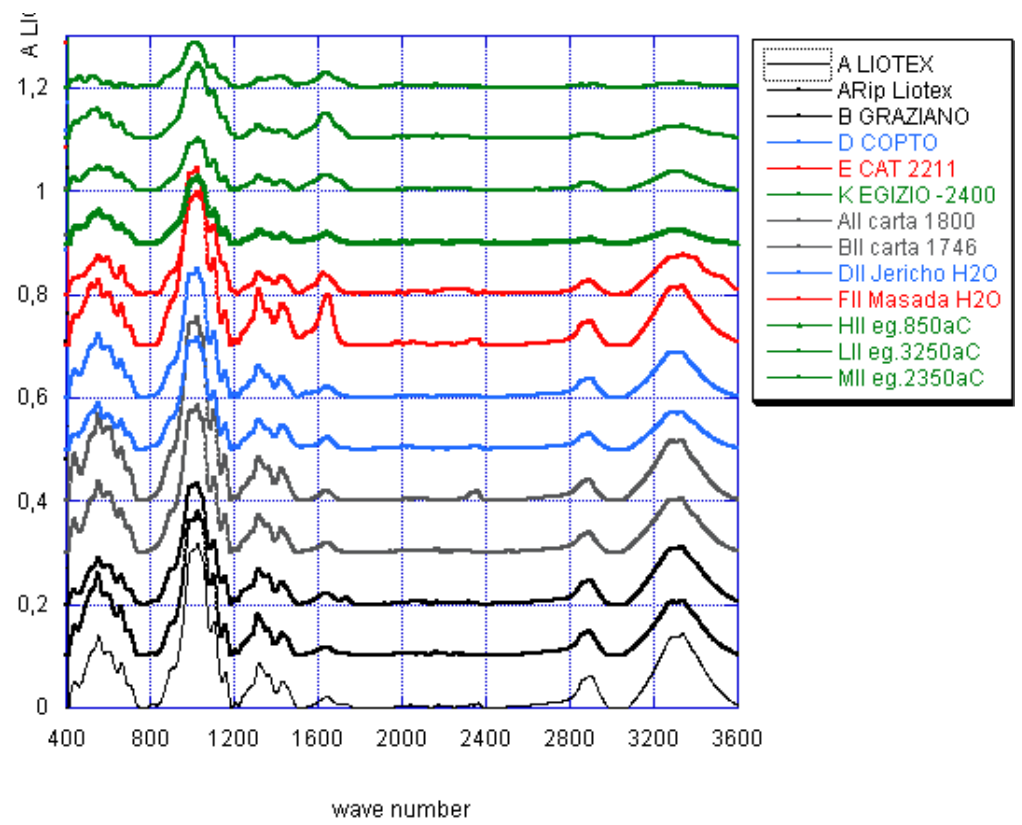

Figure 1. FT-IR ATR Absorbance spectra of the samples under analysis (baseline corrected) (from Ref. [14], with permission).

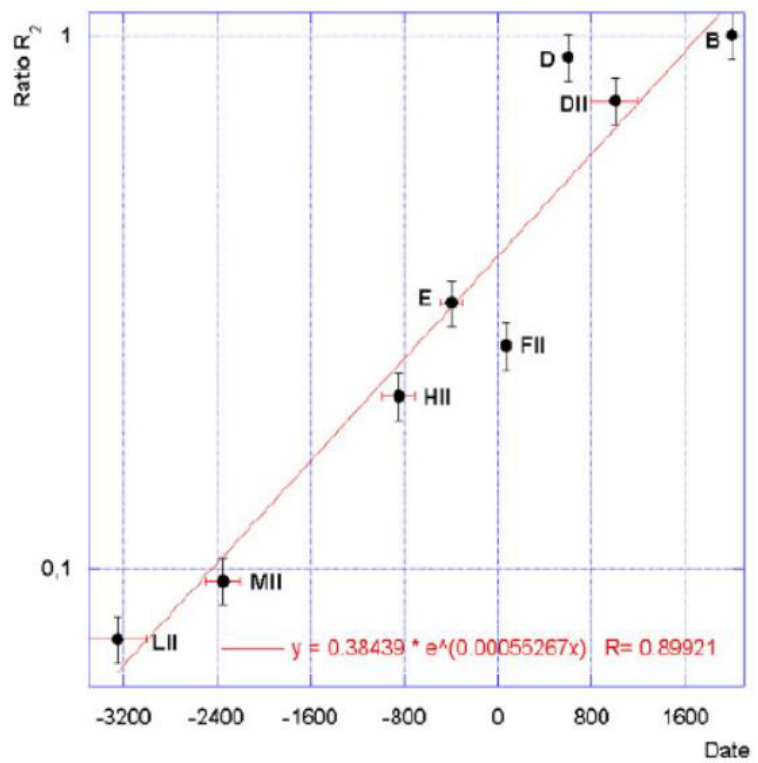

Figure 2. $\mathrm{R}_{1}$ plot of normalized data from FT-IR spectra (from Ref. [14], with permission). 


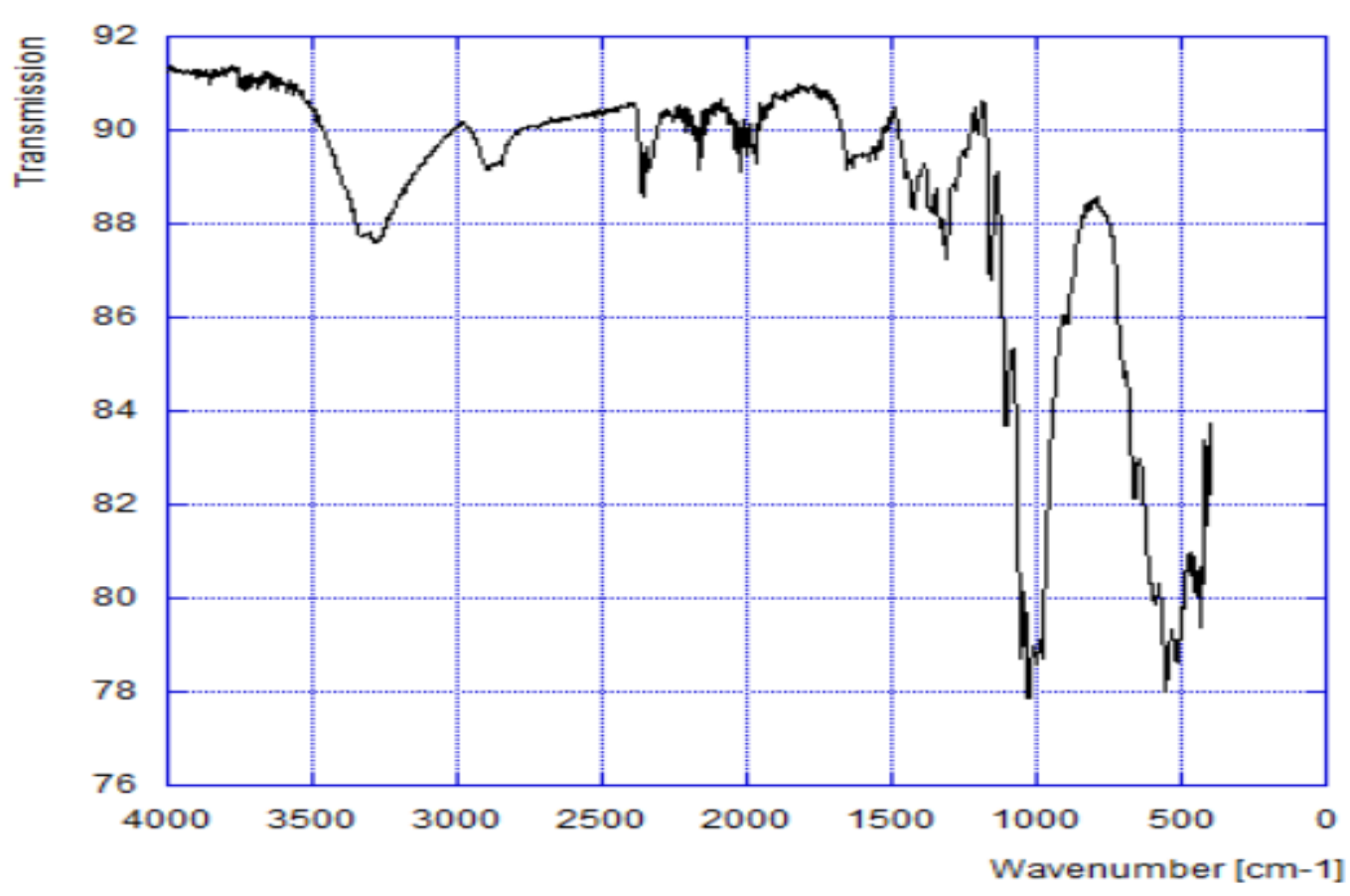

Figure 3. FT-IR/ATR spectrum obtained from a sample coming from the Shroud of Turin.

The second method is based on FT-Raman spectra (Fig. 4), and originally considered five band intensities, but finally used two of them because they appeared the most significant for a correlation with age. The band intensity relative to the $\mathrm{C}-\mathrm{O}-\mathrm{C}$ symmetric stretching mode of glycosidic linkage at $1097 \mathrm{~cm}^{-1}$ is the most significant to acquire information about flax degradation with time; furthermore, the hydrogen-bonded $\mathrm{C}-\mathrm{OH}$ band at $3251 \mathrm{~cm}^{-1}$ is quite stable with time. The ratio $\mathrm{R}_{\mathrm{COC} / \mathrm{OH}}$ of the amplitude peaks for each sample has been evaluated from the corresponding spectra (Fig. 4), where the measured Raman intensity was corrected for its baseline.

Among the reference flax samples tested, 12 were used for calibration and 3 for testing the fire effects. The fire effects have been estimated to be lower than the uncertainty and therefore negligible. The data of the samples under test are reported in Fig. 5, where two groups of samples are reported on the basis of their fluorescence ratio (lower or higher than 1.50, respectively), because this effect is not negligible.

The reference flax samples of Fig. 4 were used to determine a calibration curve; the data of the historic samples under test are reported in Fig. 5. In agreement with Ref. [14] the ratio $R_{r}$ of the amplitude peaks of each sample have been evaluated in the corresponding spectra as a function of the wavenumber $\mathrm{n}$ expressed in $\mathrm{cm}^{-1}$ :

$$
\mathrm{R}_{\mathrm{r}}=\frac{I_{1096}}{I_{3251}}
$$

Two calibration curves were defined as a function of different fluorescence values in Fig. 5. The date $\mathrm{D}$ can be then evaluated [14]:

$$
D=2451+2299 \ln R_{r} \quad \text { or } \quad D=7498+4871 \ln R_{r}
$$


respectively, in the case of low and high fluorescence (mean fluorescence values 0.786 and 1.825, respectively).

In the case of the Shroud of Turin [15], the Raman spectrum is reported in Fig. 6 and $R_{r}=0.233$ was obtained from it, giving a date 200 B.C. \pm 500 [7].

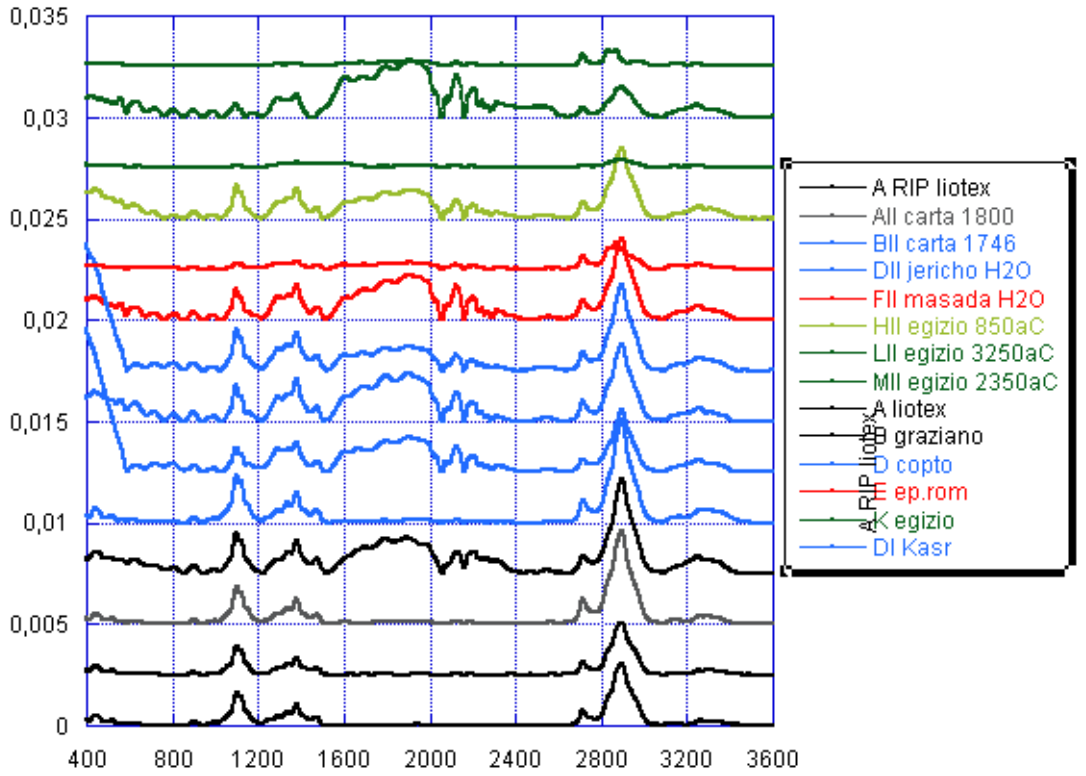

Figure 4. Raman spectra corrected for baseline of the samples analyzed (from Ref. [14], with permission).

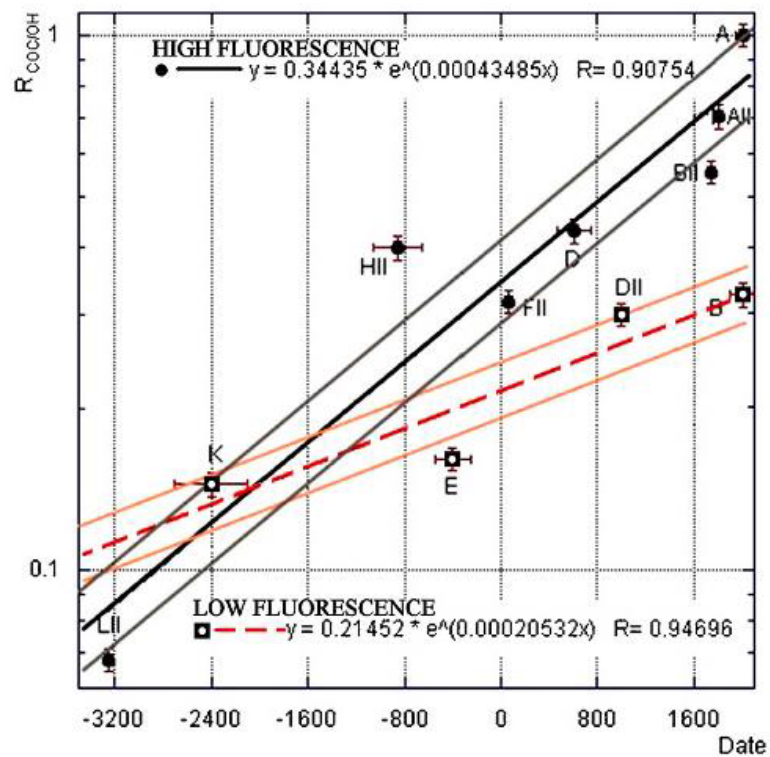

Figure 5. $\mathrm{R}_{\mathrm{COCOH}}$ plot for high (red) and low fluorescence (black) (from Ref. [14], with permission). 


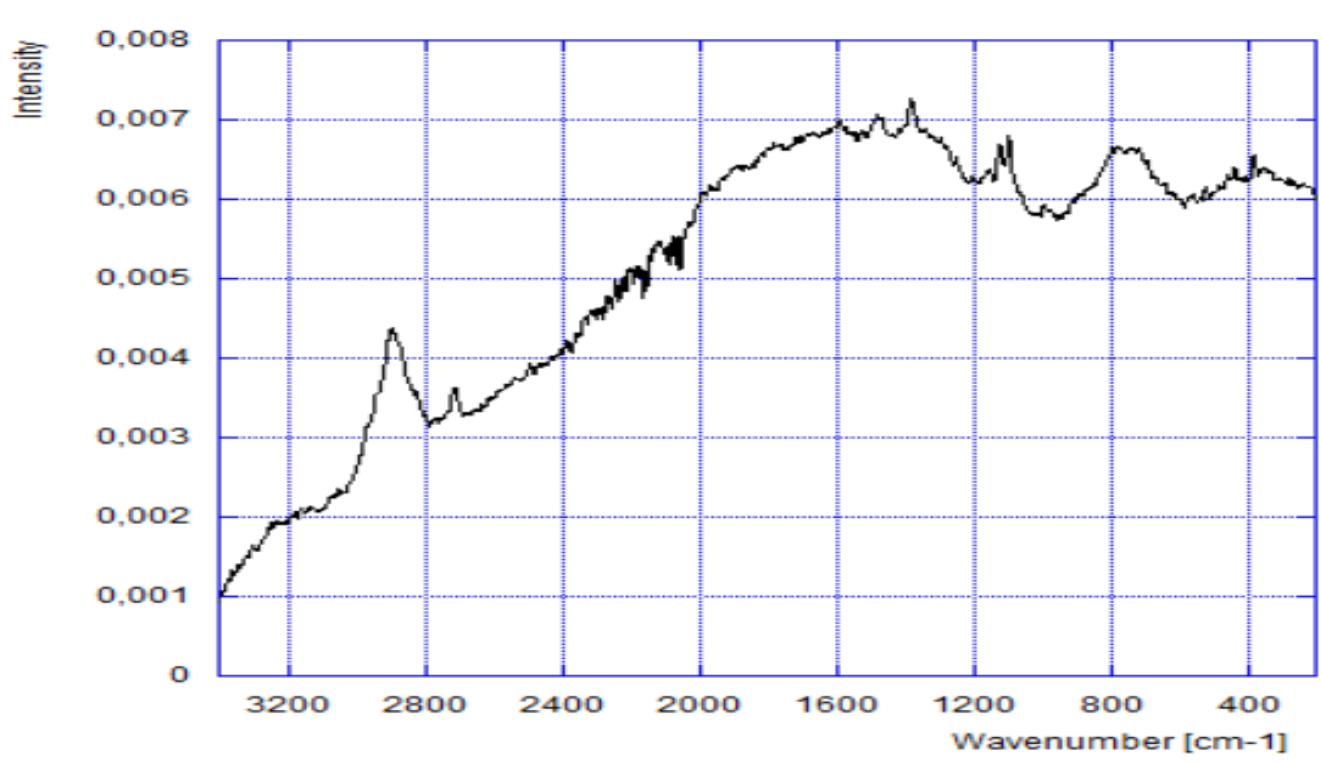

Figure 6. FT-IR/ATR spectrum obtained from a sample coming from the Shroud of Turin.

\section{Conclusive remarks}

Between the methods employed, FT-IR spectroscopy is a promising method. The intensity ratios of significant absorption regions (or their integrals in proper wavelength ranges) of the resulting spectra can properly be combined thereby obtaining a relationship (Eq. 2) characterized by a Pearson's correlation coefficient greater than 0.96 but, in case of fire effect, a bias of some centuries can be observed.

Raman spectroscopy is also a promising method if fluorescence, still present in some samples excited at $1064 \mathrm{~nm}$, is taken into account. A Pearson's correlation coefficient of only about 0.83 can be reached if fluorescence is neglected, but coefficients up to 0.95 have been obtained if this effect is taken into account. In this case it has been necessary to determine at least two different curves, and in the present case they have been referred to mean fluorescence values of 0.786 and 1.825 , respectively. Differently form IR, for Raman spectroscopy fire effects have been estimated to be lower than the uncertainty and therefore negligible.

While at present FT-IR spectroscopy can be preferred because it is not affected by the fluorescence problem, Raman spectroscopy has the important advantage to be less sensible to environmental factors and to the effects of chemical treatments of flax, such as bleaching. In fact in the present study eleven samples have been used for Raman analysis, while only eight remained for the FT-IR test after the preliminary selection. These effects should be the subject of future investigations.

The dating method was applied to samples coming from the Shroud of Turin furnishing a date of 300 B.C. \pm 400 years and 200 B.C. \pm 500 with a $95 \%$ confidence level, respectively, in the case of FTIR/ATR and Raman spectroscopy.

\section{References}

1. F.W. Langkilde, A. Svantesson, J. Pharm. Biomed. Anal. 13 (1995) 409-14.

2. Q. Shen, H. Rahiala, J. B. Rosenholm, J. Colloid Interface Sci. 206 (1998) 558-68.

3. H.G.M. Edwards, N.F. Nikhassan, D.W. Farwell, P. Garside, P. Wyeth, J. Raman Spectrosc. 37 (2006), 1193-2000.

4. K. Kavkler, A. Demsar, Spectrochim. Acta 78A (2011) 740-46. 
6. L. M. Proniewicz, C. Paluszkiewicz, A. Weselucha-Birczynska, A. Baranski, D. Dutka, J. Mol. Struct. 614 (2002) 345-53.

7. G. Fanti, P. Malfi, "The Shroud of Turin. First century after Christ", Pan Stanford Publishing Pte. Ltd., Singapore, 2015

8. K. Schenzel, A. Jahn, P. Peetla, S. Kovur Kumar, H. Ding, Pflanzenbauwissenschaften 11 (2007) 16-21.

9. R.G. Zhbankov, S.P. Firsov, D.K. Buslov, N.A. Nnikonenko, M.K. Marchewka, H. Rataiczak, J. Mol. Struct. 614 (2002) 117-25.

10. Etzion, R. Linker, U.Y. Cogan, I. Shmulevich, J. Dairy Sci. 87 (2004) 2779-88.

11. C. B. Ramsey, Archaeometry 50 (2008) 249-275.

12. F. Z. Zakaria, J. Mihaly, I. Sajò, R. Katona, L. Haiba, F.A. Aziz, J. Mink, J. Raman Spectrosc. 39 (2008) 1204-9.

13. M. Szymanska-Chargot, J. Cybulska, A. Zdunek, Sensors 11 (2011) 5543-5560.

14. G. Fanti, P. Baraldi, R. Basso, and A. Tinti, Non-destructive dating of ancient flax textiles by means of vibrational spectroscopy. Vibrational Spectroscopy 67 (2013) 61-70.

15. G. Fanti, F. Crosilla, P. Malfi, "Mechanical and opto-chemical dating of the Turin Shroud", MATEC Web of Conference, in publication. 